\title{
Perforation palatine suite à une intubation oro-trachéale prolongée : présentation d'un cas
}

\author{
Bémer J, Guillet J, Phulpin B, Maire F \\ Service d'Odontologie, Centre Alexis Vautrin, Vandoeuvre-lès-Nancy, France \\ j.bemer@nancy.fnclcc.fr
}

Un homme de 70 ans, édenté total et porteur de prothèses amovibles complètes, a été adressé à la consultation ORL du CHU de Nancy en raison d'une suspicion de perforation palatine.

Le patient présente de lourds antécédents médicaux et chirurgicaux; la dernière intervention subie s'étant compliquée de pneumopathies à répétition, elle a nécessité une intubation oro-trachéale prolongée.

L'examen clinique révèle une perforation localisée, intéressant le voile du palais, en arrière de la jonction palais dur - palais mou. L'exploration et les examens complémentaires n'objectivent pas de pathologie tumorale. L'étiologie de cette perforation est donc vraisemblablement d'origine traumatique et secondaire à l'intubation prolongée. Elle a pour conséquence une communication bucco-pharyngée entraînant une voix nasonnée ainsi qu'un reflux nasal de l'alimentation liquide et semi-liquide. En raison de l'état général du patient, une fermeture chirurgicale n'est pas envisageable dans l'immédiat. Il est alors orienté vers le Service d'Odontologie du Centre Alexis Vautrin pour une prise en charge prothétique. Un obturateur vélaire en résine, solidaire de sa prothèse amovible supérieure, a été conçu. Les résultats esthétique et fonctionnel sont satisfaisants. La perforation palatine avec communication bucco-pharyngée reste rare en dehors du contexte de la chirurgie carcinologique, et l'étiologie traumatique secondaire à une intubation oro-trachéale prolongée l'est encore davantage. Cependant, elle constitue un véritable handicap fonctionnel pour les patients. La solution prothétique retenue dans le cas présent offre l'avantage d'être non invasive, rapide à mettre en œuvre, efficace et évolutive. Elle peut donc être transitoire ou constituer une bonne alternative à la chirurgie dans les cas où celle-ci s'avère impossible. 